\title{
Testing Suicide Risk Prediction Algorithms Using Phone Measurements With Patients in Acute Mental Health Settings: Feasibility Study
}

Alina Haines-Delmont ${ }^{1}$, BA, BSc, MSc, PhD; Gurdit Chahal ${ }^{2}$, BA, MSc; Ashley Jane Bruen ${ }^{3}$, BSc, MSc; Abbie Wall ${ }^{3}$, BSc, MSc; Christina Tara Khan ${ }^{4}, \mathrm{MD}, \mathrm{PhD}$; Ramesh Sadashiv ${ }^{2}$, BE, MSc; David Fearnley ${ }^{5}$, MBChB, FRCP, MSc, MBA

\author{
${ }^{1}$ Faculty of Health, Psychology and Social Care, Manchester Metropolitan University, Manchester, United Kingdom \\ ${ }^{2}$ CLARA Labs, CLARA Analytics, Santa Clara, CA, United States \\ ${ }^{3}$ University of Liverpool, Health Services Research, Liverpool, United Kingdom \\ ${ }^{4}$ Stanford University School of Medicine, Stanford, CA, United States \\ ${ }^{5}$ Mersey Care NHS Foundation Trust, Prescot, United Kingdom
}

\section{Corresponding Author:}

Alina Haines-Delmont, BA, BSc, MSc, PhD

Faculty of Health, Psychology and Social Care

Manchester Metropolitan University

Brooks Building, Nursing Department

53 Bonsall Street

Manchester, M15 6GX

United Kingdom

Phone: 4401612472461 ext 2461

Email: a.haines@mmu.ac.uk

\section{Abstract}

Background: Digital phenotyping and machine learning are currently being used to augment or even replace traditional analytic procedures in many domains, including health care. Given the heavy reliance on smartphones and mobile devices around the world, this readily available source of data is an important and highly underutilized source that has the potential to improve mental health risk prediction and prevention and advance mental health globally.

Objective: This study aimed to apply machine learning in an acute mental health setting for suicide risk prediction. This study uses a nascent approach, adding to existing knowledge by using data collected through a smartphone in place of clinical data, which have typically been collected from health care records.

Methods: We created a smartphone app called Strength Within Me, which was linked to Fitbit, Apple Health kit, and Facebook, to collect salient clinical information such as sleep behavior and mood, step frequency and count, and engagement patterns with the phone from a cohort of inpatients with acute mental health $(n=66)$. In addition, clinical research interviews were used to assess mood, sleep, and suicide risk. Multiple machine learning algorithms were tested to determine the best fit.

Results: K-nearest neighbors $(\mathrm{KNN}$; $\mathrm{k}=2)$ with uniform weighting and the Euclidean distance metric emerged as the most promising algorithm, with $68 \%$ mean accuracy (averaged over 10,000 simulations of splitting the training and testing data via 10 -fold cross-validation) and an average area under the curve of 0.65 . We applied a combined $5 \times 2 F$ test to test the model performance of KNN against the baseline classifier that guesses training majority, random forest, support vector machine and logistic regression, and achieved $F$ statistics of $10.7(P=.009)$ and $17.6(P=.003)$ for training majority and random forest, respectively, rejecting the null of performance being the same. Therefore, we have taken the first steps in prototyping a system that could continuously and accurately assess the risk of suicide via mobile devices.

Conclusions: Predicting for suicidality is an underaddressed area of research to which this paper makes a useful contribution. This is part of the first generation of studies to suggest that it is feasible to utilize smartphone-generated user input and passive sensor data to generate a risk algorithm among inpatients at suicide risk. The model reveals fair concordance between phone-derived and research-generated clinical data, and with iterative development, it has the potential for accurate discriminant risk prediction. However, although full automation and independence of clinical judgment or input would be a worthy development for those 
individuals who are less likely to access specialist mental health services, and for providing a timely response in a crisis situation, the ethical and legal implications of such advances in the field of psychiatry need to be acknowledged.

(JMIR Mhealth Uhealth 2020;8(6):e15901) doi: 10.2196/15901

\section{KEYWORDS}

suicide; suicidal ideation; smartphone; cell phone; machine learning; nearest neighbor algorithm; digital phenotyping

\section{Introduction}

\section{Background}

Limitations in scalability, accuracy, and consistency with respect to traditional methods of predicting suicidal behavior have been recognized in the literature and meta-analyses [1-5]. Suicidality has been defined as any suicide-related behavior, including completing or attempting suicide (intent), suicidal ideation (thoughts), or communications [6]. Not everyone who experiences suicidal ideation attempts suicide, but suicidal thoughts have been shown to be linked to a higher risk of death by suicide [7]. Although some people communicate their suicidal thoughts or plans to friends and family before suicide, others do not disclose their intent [8-10]. In addition, some individuals might not seek help during a time of crisis because of various perceived constraints, including fear of stigma or disclosure, lack of time, access to services, and preference for informal help [11]. Our ability to predict suicide is limited by our understanding of suicidal thoughts and their nature [12].

\section{Suicidal Ideation, Smartphone apps, and Machine Learning}

Advances in smartphones and connected sensors (wearables) have opened new possibilities for real-time, context-related monitoring of suicidal thoughts and suicidal risk [13], for example, ecological momentary assessments [14] that allow self-reporting of suicidal thoughts as they occur in an individual's day-to-day life, naturalistic setting [15] and digital phenotyping that enables access to real-time classification and quantification of human behavior [16-18].

The use of computational data-driven methodologies that use social media to understand health-related issues (infodemiology, infoveillance $[19,20]$ ) and data mining techniques (artificial intelligence, machine learning algorithms [21]) provides additional potential in expanding our understanding of people's thoughts, feelings, behavior, etc and improving monitoring of suicide risk in real time. Although in its infancy, new research exploring suicidal ideation has shown that social media (eg, Twitter and Facebook) could potentially be used as a suicide prevention tool [10,22-26]. One study, for example, demonstrated the utility of social media blog post analysis in classifying individuals with high suicide risk in China [27]. Some research indicates that by analyzing certain patterns of smartphone use, changes in mental health symptoms could be identified [28].

Although standardized clinical tools can help to classify factors that contribute to suicide risk and understand biological markers related to suicide (trait analyses), computer science and machine learning can provide additional and timely tools to understand linguistic markers of suicide thought (state analyses) [29]. New statistical methods have been proposed and tested to achieve more accurate predictions of risk, for example, support vector machines (SVMs), deep neural nets, and random forests [13]. Evidence suggests that these methods, especially elastic net, perform better than traditional logistic regression techniques [30]. There is a shift toward developing more personalized risk profiles and using decision tree techniques that explore hundreds of predictors rather than a few clinically relevant risk factors [31]. Modern machine learning techniques are better placed to identify complex relationships between large datasets and suicide risk [13].

Early evidence generated by a pilot study using data from 144 patients with mood disorders suggests that machine learning algorithms using previous clinical data were successful in distinguishing between people that attempt suicide and those who do not, with a prediction accuracy between $65 \%$ and $72 \%$ [32].

Although there has been a growing body of research seeking to augment or advance traditional methods with the aid of machine learning in clinical psychiatry [2,4,29,30,33-37], the majority of studies rely on applying algorithms that learn from clinical data such as health care and electronic medical records, unstructured notes by providers and caretakers, or some other data carefully gathered by health care professionals.

\section{Objectives}

In this feasibility study, we aim to add to existing knowledge by using a nascent approach combining clinical data with proxy risk active and passive data collected from mobile devices to develop our algorithm. We developed a software platform to collect data on inpatients in acute mental health settings via our own mobile app, Strength Within Me (SWiM); a smartphone (iPhone); a wrist wearable (Fitbit; Fitbit, Inc); and questionnaires administered by the research team. Active risk data-patient-facing user interface modules (eg, journaling, safety plan, and mood meter) - and passive risk data that did not require direct interaction from the patient (eg, sleep monitoring) were collected behind the scenes. This information was then used to construct and train machine learning algorithms seeking to produce a risk score that deduces the likelihood of suicide. We used the risk level from the Columbia-suicide severity rating scale (C-SSRS) [38], which was assessed by mental health researchers as our standard classification target. C-SSRS is currently considered the gold standard approach for the measurement of suicidal ideation and behavior in clinical trials [39]. Previous research has confirmed the validity of the scale and its prediction accuracy for short-term risk of suicidal behavior in clinical and research settings. Studies have demonstrated that individuals who meet the criteria of high risk following the administration of C-SSRS are almost 4 times more 
likely to attempt suicide within 24 months [38]. The C-SSRS was then compared with data from proxies for risk factors [40] such as sleep quality and emotional health collected via Fitbit () trackers and the SWiM app that patients interacted with for a week during their admission.

\section{Methods}

\section{Participants and Clinical Setting}

In this phase 1 feasibility study, we collected data from service users admitted to 6 acute adult mental health wards within a National Health Service trust in the North West of England, United Kingdom. Service users who had been admitted to a ward within the last 7-10 days were assessed by nursing staff to determine study eligibility. Following informed consent, participants were given a study iPhone and Fitbit to enable use of the SWiM app and monitor their sleep and daily activity for up to 7 days. Participants were then involved in 3 interviews at three different time points (ie, as soon as possible following admission, 3 days later and 7 days later or at discharge, whatever came first) to complete a battery of assessments, including the C-SSRS [38], examining suicidal thoughts and behavior. The interviews were completed by 2 experienced researchers who were trained to administer the clinical assessments. If suicidal risk was highlighted during the interview, nursing staff were informed and an agreed protocol was followed to ensure safety. Participants were given vouchers following the completion of assessments. In total, 80 patients out of the 186 eligible consented to participate and 66 were included in the analysis based on the completion of at least two follow-up clinical assessments. This represents a $43 \%$ response rate and $83 \%$ completion rate. For a breakdown of participants, see Figure 1.

Figure 1. Strength Within Me study flow diagram. Timeframe for recruitment: January-November 2018. Included in the analysis: participants who completed C-SSRS at second follow-up. C-SSRS: Columbia Suicide Severity Rating Scale.

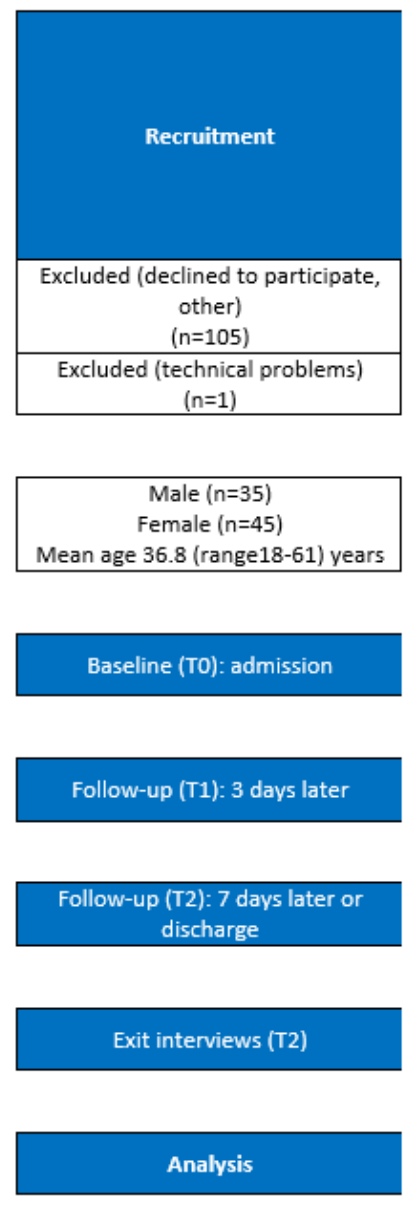

The authors assert that all procedures contributing to this work comply with the ethical standards of the relevant national and institutional committees on human experimentation and with the Helsinki Declaration of 1975, as revised in 2008. All procedures involving human subjects, ie, patients were approved by the Health Research Authority in England and the North West-Liverpool East Research Ethics Committee (Reference 17/NW/0173). Written informed consent was obtained from all inpatients.

\section{Overview of Participant Data Fed Into the Modeling Process}

At a high level, the data are segmented into (1) data entered by the user into the SWiM app; (2) data collected passively by the SWiM app, the Fitbit wearable, and the Apple Health app; (3) data directly gathered by our researchers; and (4) social interaction data for those who gave permission. We gathered a total of 173 variables - a mix of raw data such as counts of the number of journal entries and derived values that involve summary statistics or other variations of the data (eg, adding 
up minute-wise sleep records to get total sleep time or the number of interruptions in sleep). Social interaction data were excluded from the analysis because of the low response rate, that is, 8 out of the 80 participants gave permission or had access to Facebook.

As shown in Table 1, user-inputted data included participant mood, free-form journal entries, steps for personal safety plans, and custom reminders they could have set for themselves. From these entries, we collected descriptive statistics such as average mood reported (Likert Scale 1-5), average character limit, maxima, minima, and raw counts. A particular derived variable of interest from journaling was the average sentiment derived for each journal entry. This sentiment (ranging from -1 for negative to +1 for positive) was calculated via a third-party model known as the Valence Aware Dictionary and Sentiment Reasoner [41], which is catered to sentiments expressed in social media but has proven itself in other domains. The idea behind using this model was to obtain a proxy for the indication of feelings by users as they write and reflect.

Data collected by the research team included sociodemographic information, such as age and gender, and clinical assessment data. The key information that we used in the modeling was the researchers' assessment of the patient through the C-SSRS, which was assessed a maximum of 3 times (patient entry, 1 or two follow-ups during their hospital stay, and exit). All 80 consenting users were at risk upon entry to the ward (when the first test was done), so at this point, no prediction was done. The initial thought was to compare results against an intermediate survey result and exit survey result, considering the change in risk, but we did not have enough exit surveys for 2 different time period comparisons. Overall, 66 out of the 80 participants had taken at least a second survey where risk level was reassessed, and that was the population included for prediction. There was a 3-7 day wait from the first assessment to the second assessment.

Finally, we included passive data gathered via the phone and the Fitbit wearable, such as details about a user's step frequency and count from Apple's Health kit app, minute-level sleep data from Fitbit, and engagement patterns with the phone (eg, number of log-ins to the SWiM and the number of times a certain section was visited). Levels of engagement with study data are presented in Table 2.

Table 1. Strength Within Me study data.

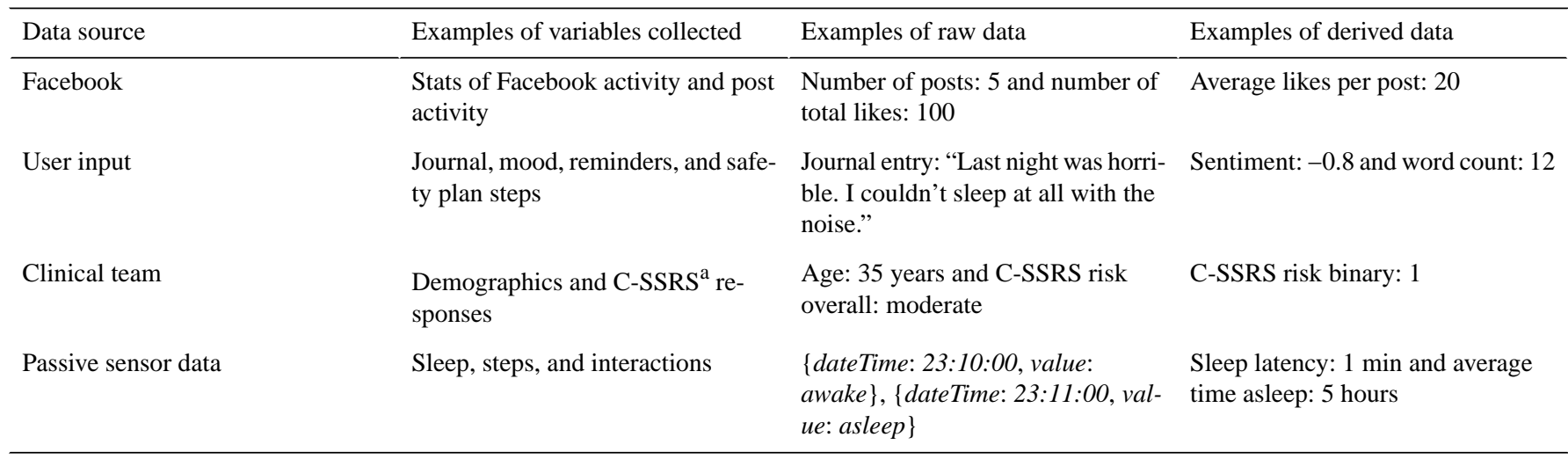

${ }^{\mathrm{a} C}$-SSRS: Columbia-suicide severity rating scale.

Table 2. Engagement rate across active and passive data in the study ( $\mathrm{N}=66)$.

\begin{tabular}{ll}
\hline Data source & Rate, $\mathrm{n}(\%)$ \\
\hline Step-related features (Fitbit and iPhone) & $26(40)$ \\
Journal entries (self-documented via SWiM ${ }^{\mathrm{a}}$ app) & $45(68)$ \\
Mood entries (self-reported via SWiM app) & $53(80)$ \\
Phone activity (data usage) & $66(100)$ \\
Sleep (Fitbit) & $59(90)$ \\
\hline
\end{tabular}

${ }^{\text {a }}$ SWiM: Strength Within Me.

\section{Modeling}

\section{Machine Learning Setup and Data Analysis in Our Clinical Setting}

As a first step toward developing an algorithmic risk score that is valid in predicting suicide risk, we framed the problem as a supervised, a binary classification problem in which users were categorized in terms of levels of risk of low risk versus high risk using the information specified above. These low risk and high risk labels were derived from the overall C-SSRS risk scores obtained after asking participants a range of questions on previous attempts, ideation, etc. Usually the 3 categories are low, moderate, and high, but we grouped moderate and high for the sake of tractability from a modeling perspective. From a machine learning perspective, this aids in what is commonly referred to as the class imbalance problem [42], where certain 
categories have relatively few labels to their other counterparts. This makes it statistically more difficult to identify, and these categories as models are inclined to achieve high scores by predicting the most common class; we turned a distribution of 36 low, 5 moderate, and 25 high to 36 low and 30 high. Choosing a binary case was helpful in dealing with the class imbalance issue, as models are data-dependent in terms of volume (ie, the more examples, the better job they do in learning). This is especially critical when we take into account the limitations in our data; to fairly judge the model performance, we must partition the data (a test set and training set via k-fold cross-validation [43]) to assess how well the model can predict risk on new users given what it is learned from old users [44]. From a risk-app perspective, although it would be ideal to place users on a continuum of risk levels, it is critical to first assess the feasibility of identifying users at discrete thresholds as well as seeing the degree to which we can match the current standard in risk assessment.

Our low risk and high risk categories were mapped to binary outputs of 0 or 1 . Some features derived from a user's journal entry are the word length and sentiment score (ranging from negative with -1 to positive +1 ; for further information, please refer to the source model from which this is derived [41]). To account for the time dependency in the data (multiple journal entries across multiple days for example), a majority of the features engineered were done so in a summary statistics fashion (mean, median, variance, etc). For example, the average journal word count per day over the user's total number of entries was used to summarize one aspect of a user's journaling behavior over their time with the app.

We curated 172 features formulated from categories of sleep data, journal entries, data usage, mood, and app activity statistics. For more information, a comma-separated values file including the full list of features incorporated into modeling (besides uid, which is user id to anonymize yet identify patient) is included in Multimedia Appendix 1. The 172 features were projected down to a 5-dimensional space by principal component analysis (PCA). This sample provides an insight into replicability. Any feature that has a summary statistic attached such as mean or std was done over the course of the 3-5 days before the second assessment. Categorical features such as gender were mapped to numerical (in this case binary) outputs for the algorithm to consume.

This is typically considered a relatively high number of features relative to the amount of possible supporting data points per number of users recorded. To provide a more suitable set from which a machine learning algorithm may distinguish a signal for risk, we turned to feature selection and dimensionality reduction techniques. Our aim was to cut down to a smaller set of features that may also be interpretable and grounded in clinical knowledge of risk factors. We, therefore, opted for PCA [45] as our dimension reduction technique and used Random Forest [46-48] to help in terms of feature selection as well to check the reliability of our reduction. Algorithms such as SVMs [49] are designed in such a manner as to overcome dimensionality issues, but they were experimentally confirmed to be unsuited to the task due to the size of the data.

For our study, the random forest model was composed of 25 decision trees. We took a look at the top 30 of the 170 original features and found that journal-related features such as average feeling, cell activity such as the variation in user's data usage, sleep-related features such as average sleep efficiency (time spent sleeping or total time spent in bed), and other natural indicators, mostly known to clinical psychology, are markers of risk. For an example of a decision tree formed for our data, see Figure 2. The tree is read similarly to a flow chart in a top-down, left-right fashion. For example, at the top, we start with an entropy of 0.997 (entropy of 1 means complete uncertainty with 0 as certainty) [19-21], as we have 25 people in the low-risk category and 22 in the high-risk category. We, then, look at their average journal feeling, and if it is less than 3.161 , we go to the left node with a subgroup of 34 people, otherwise the right node with a subgroup of 13 people. Following the right node, we now have a subgroup of 13 people with an average journal feeling greater than 3.161. On the basis of this characteristic alone, we reduce entropy to 0.619 (we are more certain of our group) and have 11 users correctly identified as high-risk, but 2 low-risk users misclassified as high-risk users. Reduce misclassification: we again split by the average amount of time the user has spent in bed. If they have greater than 541 min spent in bed in a day, a subgroup of 9 out of 9 people is correctly identified as high-risk users. However, we see that for less than 9 hours, we also predict high risk and have complete uncertainty (entropy 1), as the subgroup of 4 people is evenly divided among the classes. Once we reach 1 of these leaves or terminal nodes, we can read the decision process used to get there. For example, for the right-most leaf with 9 samples we discussed, users with an average journal feeling greater than 3.161, who also spend more than 9 hours in bed, are identified as high-risk users. Similar interpretations can be made for the other 6 terminal nodes. Worth noting is that the features are ordered top-bottom in terms of ability to split classes and reduce entropy; by this criterion, we see journal feeling as the most important feature, time in bed as second, and so on. 
Figure 2. Example of a decision tree formed for the Strength Within Me study data.

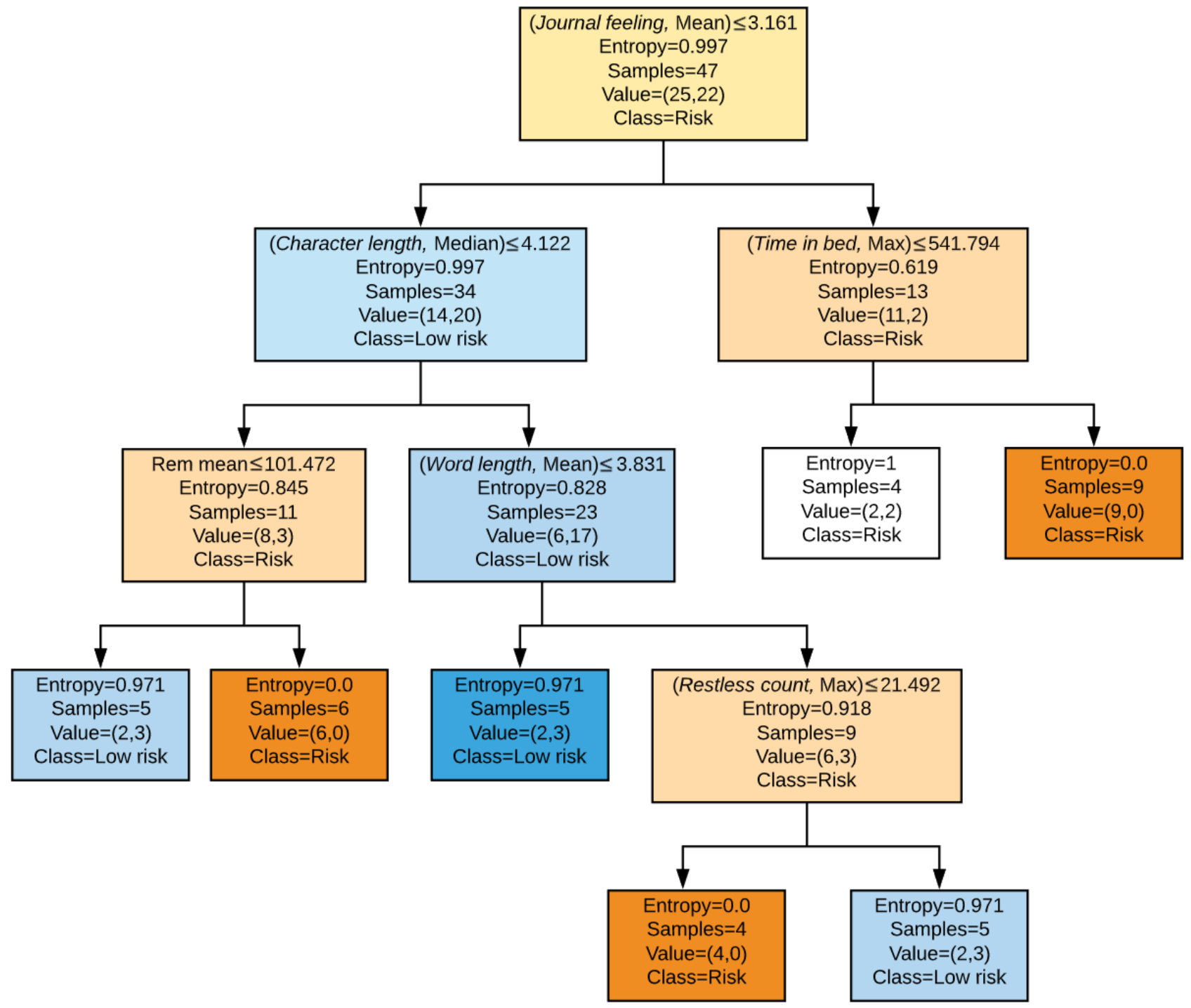

\section{Principal Component Analysis}

For dimensionality reduction and to guard against overfitting, we turned to PCA. On a high level, PCA groups features that are correlated with one another into new features (principal components) that hold the most signal in terms of variation in the data [44]. The idea is that features that explain a high level of variability found in the data produce most of the signal needed to distinguish categories. Those features which do not contribute as too strongly are discarded; by doing so, we can obtain a concise set of features at the cost of a small drop in prediction performance. Formally, PCA is an orthogonal linear transformation that maps the data to a new coordinate system such that the bulk of the variance of the projection is covered by the first $\mathrm{k}$ components, where $\mathrm{k}<$ total number of original features and components are linear combinations of the originals. Another important characteristic of PCA is that it is not optimized for class separability and may be considered as an unsupervised model. This is critical as we aim to achieve generalizations outside of the data at hand, and we do not want to overfit our final model. To provide a visualization of the PCA transformation on our data, an example of a 2-dimensional, 2 components PCA is given in Figure 3.

After looking at the variance captured up to 100 possible components, we settled for the first 5 components, as they accounted for $55 \%$ of the variance. Our first 5 components are described in Table 3, along with the themes/patterns identified after reviewing which features were grouped. We were assured that these components made sense in terms of clinical knowledge of how sleep quality, mood, activeness, and other characteristics are indicators of mental health [50-53]. Moreover, the top 30 features of our feature selection from random forest strongly overlapped with these features, and so we were further assured in terms of potential predictive power. 
Figure 3. A diagram of principal component analysis. A high-dimensional dataset has been flattened to a 2-dimensional space where the new axes correspond to the principal components (they point in the direction of the largest variance of the data).

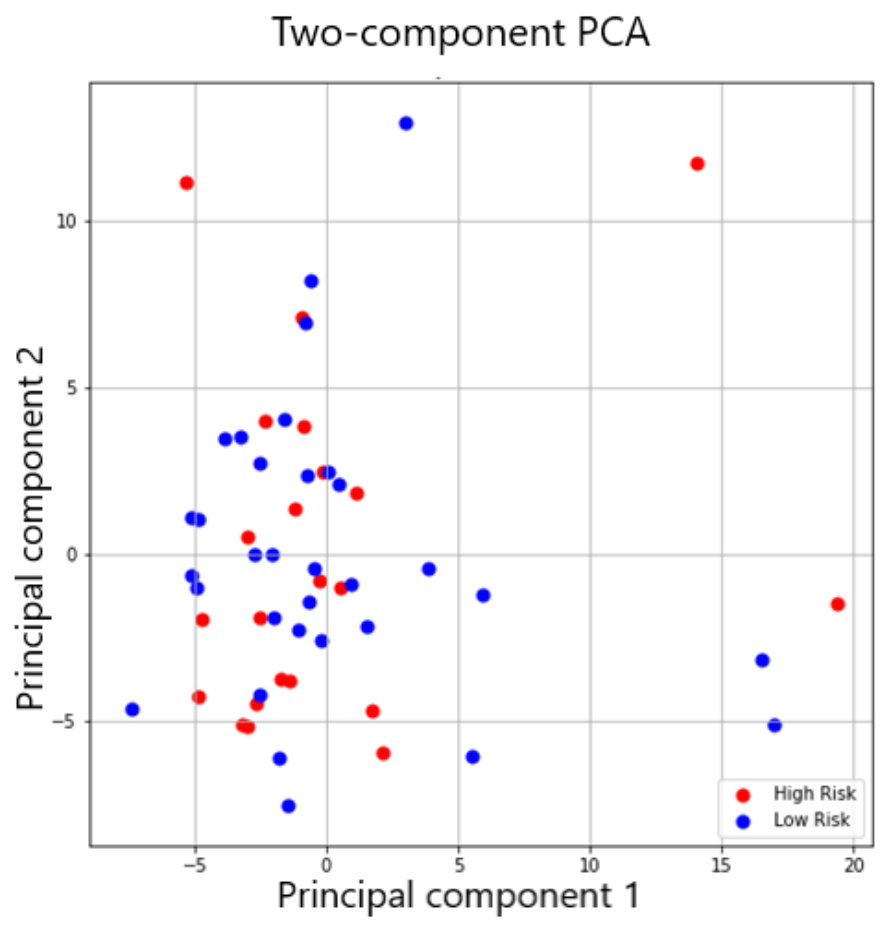

Table 3. Principal components analysis components and patterns.

\begin{tabular}{lll}
\hline Component & Description & Themes, patterns \\
\hline First component & $\begin{array}{l}\text { Maximum efficiency, average efficiency, median efficiency, max time in bed, and } \\
\text { number of sleep recordings }\end{array}$ & Ability to sleep, sleep quality \\
Second component & $\begin{array}{l}\text { Number of packets sent, number of times connected to Wi-Fi, number of times con- } \\
\text { nected to cellular data plan, and number of times journal entered }\end{array}$ & User app activity, data presence \\
Third component & $\begin{array}{l}\text { SD sleep start, median journal feeling, max sleep start, max journal feeling, minutes } \\
\text { in bed, and minimum journal feeling }\end{array}$ & Feeling versus sleep activity \\
Fourth component & $\begin{array}{l}\text { Median char length, median word length, median journal feeling, SD rest duration, } \\
\text { and max rest duration }\end{array}$ & $\begin{array}{l}\text { Journal input versus resting } \\
\text { variability }\end{array}$ \\
Fifth component & $\begin{array}{l}\text { Median sentiment, SD number of awakenings during sleep, number of awakenings } \\
\text { during sleep, and minimum sentiment }\end{array}$ & $\begin{array}{l}\text { Sleep quality and reflection } \\
\text { tone }\end{array}$ \\
\hline
\end{tabular}

\section{Results}

\section{Prediction Algorithm Testing}

We tested a series of algorithms that we thought would be best suited to predicting levels of risk from a theoretical perspective. Often referred to as the bias-variance trade-off $[44,54,55]$, there is often the case with model selection that the best model should not be too simplistic such that its crude predictions miss a bulk of the cases, nor should it be overly complex such that its high sensitivity perfectly fits the data, but fails to generalize to new, unseen data. This principle, along with other individual algorithm properties, helped guide the experimentation. As discussed in the literature [56], increasing the complexity and flexibility of a model tends to allow it to understand more nuanced relations but at the cost of being overly sensitive to noise within data and overfitting. Hence, not only were models of varying complexities chosen for comparison from linear models such as logistic regression to nonparametric models such as K-nearest neighbors (KNN), but the parameters within each model were also tuned by choosing the number of neighbors and reducing dimensionality through PCA.

It is important to mention that these models are selected and judged based on various metrics that aim to capture the objective for which the model is needed. Certain metrics also have advantages over others depending on the imbalance of classes, nature of the data (categorical or numerical), and other factors. As we had a nearly balanced dataset, and this was a feasibility study, we opted for the simplest way to measure performance, in this case accuracy, to understand metric of accuracy where we measured the number of correctly predicted observations over the total number. As a baseline, we looked at the simplest heuristic of predicting the majority class of low-risk users. This produced an average accuracy of $53 \%$. 
Random forest was tested as it is generally agreed upon as a strong out-of-the-box model that performs well on various datasets in different contexts as well as having interpretability through the feature importance it can help provide $[46,48]$. Logistic regression was another model considered due to the log-odds interpretability for the coefficients to each of the features (usually referred to as explanatory variables in explanatory contexts) and natural fit to classification problems [44]. SVMs [44,49] were also tested as they have the design of naturally combating the curse of dimensionality through the transformations they do to the data (kernel trick). SVMs are also rather sophisticated models that tend to produce near state-of-the-art results (barring neural networks which at the time of writing are highly data-hungry, and not necessarily interpretable). Finally, we considered the KNNs algorithm, which is often sought due to simplicity as well as the natural heuristic of classifying based on how close observations are to one another [57].

To test the performance, we performed k-fold cross-validation with $\mathrm{k}=10$. This means that we randomly partitioned the data into 10 pieces (folds) and used 9 of them to train the model and 1 as an unseen piece (fold) to test on. This was done such that each of the 10 folds was used as the unseen/testing data at a given iteration. The idea was to obtain the expected accuracy of a model when exposed to new data by simulating variations of data seen to unseen data. We repeated this process 10,000 times to obtain a more stable estimate, as there are many ways to partition this data into 10 folds. Table 4 summarizes the results.

Table 4. The average cross-validation accuracy, along with the SD of the accuracy observed for the various folds.

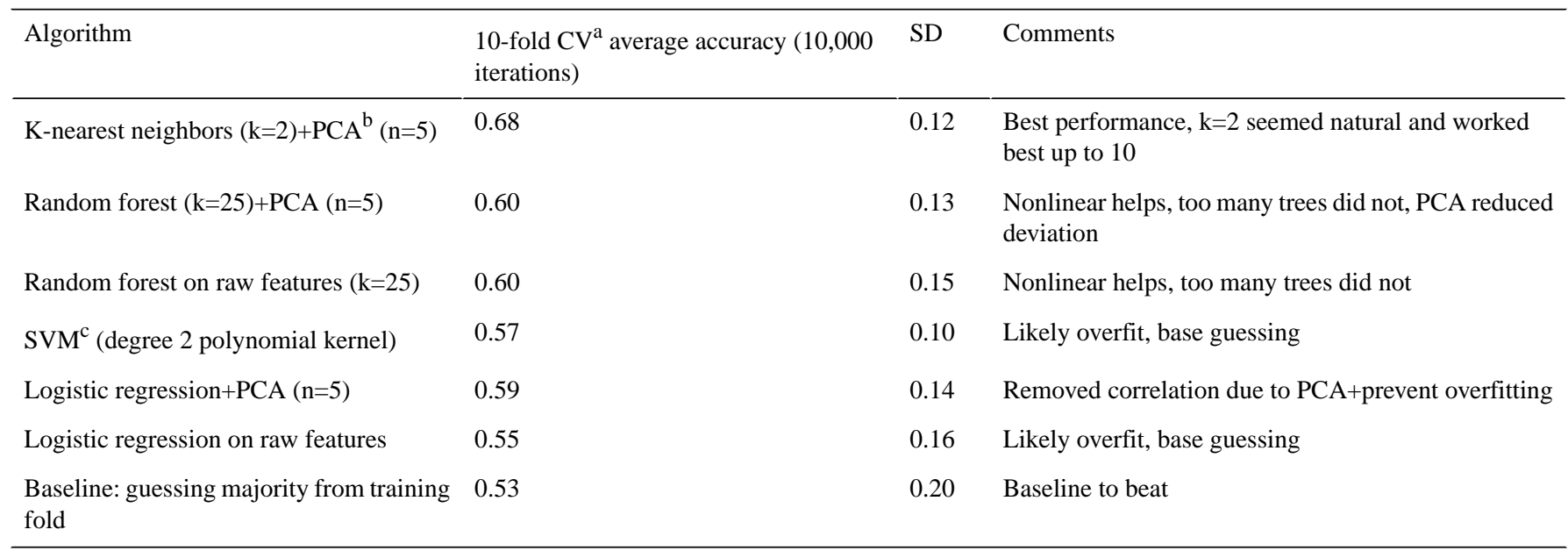

${ }^{\mathrm{a}} \mathrm{CV}$ : cross-validation.

${ }^{\mathrm{b}} \mathrm{PCA}$ : principal component analysis.

${ }^{\mathrm{c}} \mathrm{SVM}$ : support vector machine.

Logistic regression failed to perform much better than baseline. With the raw features, it performed poorly likely due to overfitting and high collinearity between some features (eg, median sleep time and mean sleep time). We removed most of this through PCA and performed slightly better on average at $59 \%$, but the SD of $14 \%$ was worrying, given its below baseline lower end (worse than majority guessing). Similarly, SVM failed to perform much better, and of the different kernels, we present the polynomial degree 2 kernel as it performs best out of other variations (higher order polynomials, radial basis kernels, and linear). We defer the explanations of these kernels to the literature. Random forest performed better than either of the other 2 algorithms, but worst-case folds still fell below baseline.

\section{The k-Nearest Neighbors Algorithm}

The most promising was the KNNs algorithm with $\mathrm{k}=2$ with the 5 principal components discussed earlier as features with not only an average accuracy of $68 \%$ (averaged over 10,000 simulations of splitting the training and testing data via 10-fold cross-validation) but with an SD of $12 \%$, which resulted in its worst performance just above baseline at $56 \%$ and upper limit of $82 \%$ (Figure 4 ). In terms of false positive and true positive rates, the model achieved an average AUC of 0.65 (Figure 5). We applied a $5 \times 2 \mathrm{cv}$ combined $F$ test to test the model performance of $\mathrm{KNN}$ against the baseline classifier that guesses training majority, random forest, and other models such as supported vector machine and logistic regression and achieved $F$ statistics of $10.7(P=.009)$ and $17.6(P=.003)$ for training majority and random forest, respectively, rejecting the null performance being the same $[58,59]$.Due to the promising results of the algorithm, we explain it to readers unfamiliar with it. The KNN algorithm essentially follows the saying of birds of the same feather flocking together. That is to say, the way prediction is performed using this algorithm is that for a new test point, the distance (usually the well-known Euclidean distance) is computed between the new point and $\mathrm{k}$ of the closest previously labeled observations. Of the $\mathrm{k}$ neighbors, the majority class is chosen as the label for the test point. For example, with $\mathrm{k}=5$, we look at the features of a new person whose risk has not been identified yet and look at 5 people with the features closest matching this new person out of the training set. If 3 of them are high risk and 2 are low risk, the new person is identified as high risk, with 3 votes to 2 . For even numbers of $k$, such as 6 , where there might be ties, we weight the votes by proximity. Therefore, with respect to our PCA features, we are comparing 
people who have similar sleep characteristics, data usage, and so on. We found $\mathrm{k}=2$ to perform best in our scenario, likely due to the low sample size as well as high variability among users.
We used a Euclidean distance metric and enforced each feature to have equal distance weighting (uniform weights).

Figure 4. Example of nearest neighbors with $\mathrm{k}=2$ with data in 2 dimensions. Here, the new test point is $\mathrm{x}$ and has 1 minus neighbor and 1 plus neighbor as its 2 closest neighbors. As the minus neighbor is closer, the new point $\mathrm{x}$ will be classified as minus. "+" stands for positive class, "-" for negative class, and " $\mathrm{x}$ " for new data point that has yet to be assigned a class.

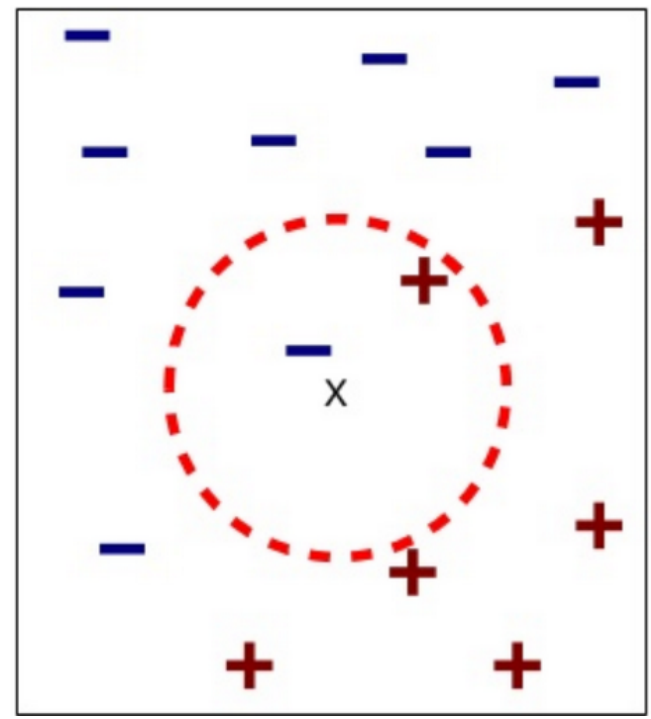

Figure 5. Receiver operating characteristic curve for k-nearest neighbors. AUC: area under the curve.

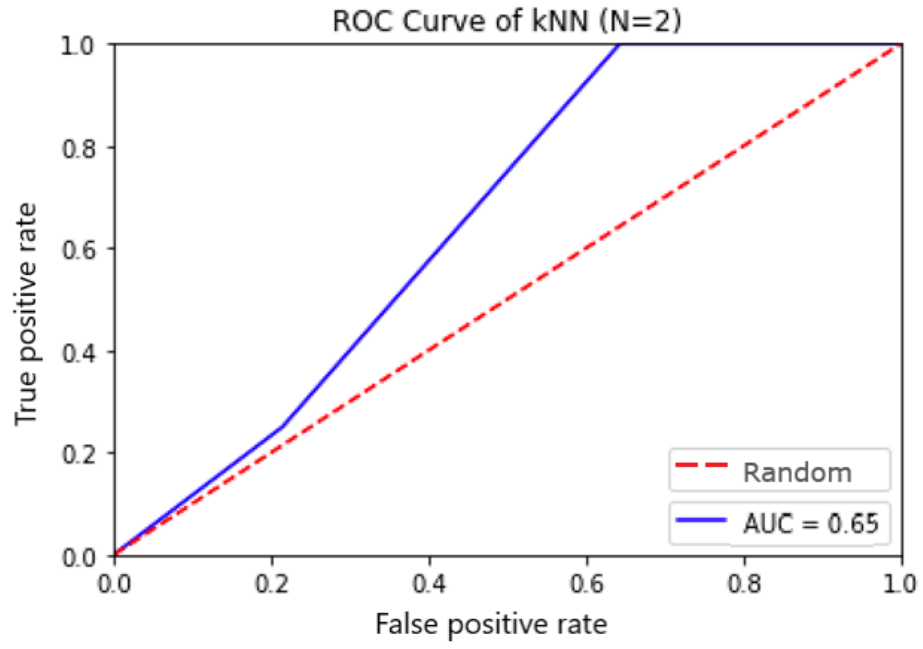

\section{Discussion}

\section{Principal Findings}

The results from this feasibility study indicate that, although not a perfect predictor, the KNN model is suitable for this study because it has shown the ability to separate users deemed at risk of suicide from the C-SSRS to those not deemed at risk at an average rate beyond just randomly guessing (ie, at an average rate $15 \%$ beyond randomly guessing the majority to be at low risk). These are early indications that it is possible to predict risk using the data collected in this feasibility study, using the KNN algorithm. The data used to inform this included users' sleeping activity, step activity, self-reported mood, journaling thoughts, and activity levels as measured by a phone app.
This is a crucial first step in automatic risk assessment, as we managed to build an algorithm that predicts suicidal risk at a rate significantly better than the baseline of guessing the simple majority that were collected directly from smartphone interactions. This is also promising, as we are working with a relatively small dataset from a machine learning perspective. This is the basis for future phases of this study, where we will be looking to test the model on additional users of mental health services for further testing of concept and generalizability.

The implications of this feasibility study are highly significant for building capacity for suicide risk prediction (future risk) or detection (real-time, current risk). With a low proportion of suicide attempters who actually access mental health services [60], it is essential to develop and test nonclinical means of assessing risk. Given the dynamic nature of suicide ideation 
and suicide risk, new methods are needed to track suicide risk in real time [61], together with a better understanding of the ways in which people communicate or express their suicidality [25]. Mobile apps could be better suited to help prevent suicide by offering support in situ and at the time of crisis [62].

Although previous studies have utilized electronic health care record data to create an actuarial model of suicide risk [30,34,35,37,63-65] or focused on a single aspect of user input such as language $[29,30,36]$, this study adds to the literature by introducing external, user-generated input, and smartphone data and combining it with clinical data. Our study adds to evidence that reports on the use of external, nonclinical data to predict suicidality. The results are promising, although we used basic, simpler, and routine biometrics (collected via iPhone and Fitbit), compared with data used in previous research. Studies aiming to predict mental state (short term) have used multiple (self-report) measurements and a wide range of bio sensors $[12,15,66]$.

\section{Strengths, Limitations, and Further Testing}

We recognize that our study is limited by the short follow-up period of up to a week; thus, future iterations would need to extend to a longer period of study to explore the time sensitivity of model predictions over varying time windows (eg, predicting current risk vs 1 week out). Short-term risk prediction is difficult because any inference is based on limited data, which means that meaningful signals are lost due to noise from highly variable behaviors [13]. There is promise in improvement as the amount of data available for training and testing increases. Previous research and machine learning literature [67-70] points to expected improvement in performance and reliability in test results as the sample size grows, particularly in this classification setting. We expect that roughly doubling the sample size would achieve more practical results where the possibility of implementation would be appropriate.

Although the results from this feasibility study have been promising in producing a signal, in terms of operationalizing risk for suicide, future steps would be moving beyond survey-generated risk scores. Before taking that leap, the intermediate step would be to further validate the algorithmic results by collecting additional, more substantial test data. Where the experiment excels in the data sources are diverse rather than strictly clinical and allow for natural extension to outpatient settings. In addition, given the probabilistic nature of the algorithm, there will naturally continue to be a trade-off between false positives and false negatives as the model improves, and hence, medical, human attention in decision making will remain critical. We propose that the algorithmic approach provides a supplement and an additional facet to clinical judgment.

Therefore, having achieved a signal from the data for risk in phase 1, phase 2 (proof of concept) will involve collecting more data to not only see if modeling improves but also to test other models such as predicting the risk score trajectory. Enforcing a minimum of 2 C-SSRS assessments, we can try to model changes in risk. We also intend to experiment with more features, particularly those involved in text mining, as most journaling features were relatively surface level. Moreover, we

aim to look at prediction stability over time, as this prediction was made within a couple of days from usage to assessment.

Our final aim is to form our own standard so as to break away from dependency on the C-SSRS, as we look to go beyond information gathered in a formal survey that depends solely on human judgment. Further research will enable us to test the viability of automation and machine learning to identify suicide risk by comparing predictions of risk with eventual outcomes as well as testing out the model in different settings and populations (eg, community).

We would also like to point out that, although mobile phones and apps are ubiquitous and have the potential to be an efficient and cost-effective approach to addressing mental health problems [71], this study indicated that there are certain costs that limit the widespread adoption of health apps within mental health services (weather inpatient and community settings). These are related to access to smartphones, connectivity, updating, and maintenance of technology. The premise for this study was that, in line with the UK population statistics indicating that approximately $95 \%$ of households own a mobile phone [72], of which a high proportion are smartphones, participants would have access to and use their own smartphones for the study. Following initial scoping, the authors realized that only a small proportion of inpatients had access to a smartphone. In addition, the SWiM app was configured (in its current testing form) to operate only with iOS products, that is, an iPhone. We cannot confirm the extent to which given participants study iPhones might have affected the results; this is something that needs to be further explored. We can, however, highlight that participants were enthusiastic about using Fitbit wearables and the Fitbit app on the phone, which may or may not have encouraged them to use the SWiM app as well.

\section{Conclusions}

Although in its early stages, research in this area suggests that using smartphones to enquire about suicidal behaviors can be a valuable approach and not a risk factor for increasing suicidal ideation [12]. Given the heavy reliance on smartphones and mobile devices around the world, this readily available source of data is an important and highly underutilized source that has good potential to improve mental health risk prediction and prevention and advance global mental health.

However, although full automation and independence of clinical judgment or input would be a worthy development for those individuals who are less likely to access specialist mental health services, and for providing a timely response in a crisis situation, we need to acknowledge the ethical and legal implications of such advances in the field of psychiatry [72,73]. The use of machine learning in suicide prediction needs a strong evidence base across different settings, populations, suicidal behaviors, and datasets, before considering full integration in health care settings. For the time being, if proven accurate and scalable, machine learning algorithms for suicide risk detection are likely to complement rather than replace clinical judgment [72]. Although smartphones provide us with opportunities to gather data on real-time dynamic risk factors for suicidal behavior, which would be almost impossible to monitor on discharge (from mental health settings), more research is needed to 
validate the utility of risk markers for suicide behavior and confirm a safe and clinically effective way to use these data to inform practice [13]. More work is needed before we can achieve safe and effective integration within mental health settings, while remaining attentive to key ethical implications. An interesting ethical dimension is related to the use of the KNN algorithm, which requires continued access to the pooled data of (at least a subset of) multiple participants to subsequently label new cases. Although testing this in a controlled setting with inpatients who have provided consent for the use of their data might be straightforward, it is uncertain if service users in the community would accept to have their suicidal trajectory data shared for this purpose or how mental health services would be able to bridge the gap. Furthermore, to achieve high accuracy in terms of short-term risk prediction, a wide variety of data from multiple sources will need to be collected, with data integration as a key component [13]. We, therefore, expect multiple data governance, privacy, and intellectual property issues at stake.

\section{Acknowledgments}

This work was supported by the Department of Health, Global Digital Exemplar (ref: CENT/DIGEX/RW4/2017-10-16/A). The authors would like to acknowledge Abhijith Nagaraja's contribution. He was the lead architect and engineer to design and deploy the iOS apps used to collect data for study participants. Abhijith was also responsible for designing and developing the backend software to ensure that data are captured and stored as per privacy policy standards requirements.

\section{Authors' Contributions}

AH has contributed to the design of the study, set up and management of the study, drafted sections of the paper, and revised it critically. GC has conducted all the data analysis and interpretation, drafted sections of the paper, and contributed to the overall revision. $\mathrm{AB}$ and $\mathrm{AW}$ collected all the data for the work and critically revised the paper. $\mathrm{CK}$ contributed to the design of the study, drafted sections of the paper, and critically revised it. RS was responsible for managing the design and development of the software app, product analytics, and delivery as well as revising the paper before submission. DF was the chief investigator for this project, having contributed to the design and oversight of the project as well as drafting sections of the paper and critically reviewing it.

\section{Conflicts of Interest}

$\mathrm{AH}, \mathrm{GC}, \mathrm{AB}, \mathrm{AW}$, and RS have nothing to disclose. CK reports grants from the Stanford University School of Medicine, during the conduct of the study, and personal fees from The Risk Authority in 2016 before the study, outside the submitted work. DF reports being a member of the Board of Managers for Innovence Augmented Intelligence Medical Systems Psychiatry, a limited liability company between Mersey Care National Health Service Foundation Trust and The Risk Authority, Stanford. This has overseen the development of technology that is undergoing evaluation in this research study.

\section{Multimedia Appendix 1}

Sample training data.

[XLSX File (Microsoft Excel File), 13 KB-Multimedia Appendix 1]

\section{References}

1. Capodanno A, Targum S. Assessment of suicide risk: some limitations in the prediction of infrequent events. J Psychosoc Nurs Ment Health Serv 1983 May;21(5):11-14. [Medline: 6552289]

2. Franklin JC, Ribeiro JD, Fox KR, Bentley KH, Kleiman EM, Huang X, et al. Risk factors for suicidal thoughts and behaviors: a meta-analysis of 50 years of research. Psychol Bull 2017 Feb;143(2):187-232. [doi: 10.1037/bul0000084] [Medline: 27841450]

3. Haney EM, O'Neil ME, Carson S, Low A, Peterson K, Denneson LM, et al. Suicide Risk Factors and Risk Assessment Tools: A Systematic Review. Scotts Valley, California: CreateSpace Independent Publishing Platform; 2012.

4. Ribeiro JD, Franklin JC, Fox KR, Bentley KH, Kleiman EM, Chang BP, et al. Self-injurious thoughts and behaviors as risk factors for future suicide ideation, attempts, and death: a meta-analysis of longitudinal studies. Psychol Med 2016 Jan;46(2):225-236 [FREE Full text] [doi: 10.1017/S0033291715001804] [Medline: 26370729]

5. Turecki G, Brent DA. Suicide and suicidal behaviour. Lancet 2016 Mar 19;387(10024):1227-1239 [FREE Full text] [doi: 10.1016/S0140-6736(15)00234-2] [Medline: 26385066]

6. Goldsmith SK, Pellmar TC, Kleinman AM, Bunney WE, editors. Reducing Suicide: A National Imperative. Washington, DC: National Academies Press; 2002.

7. McAuliffe CM. Suicidal ideation as an articulation of intent: a focus for suicide prevention? Arch Suicide Res 2002 Nov;6(4):325-338. [doi: 10.1080/13811110214524]

8. Wolk-Wasserman D. Suicidal communication of persons attempting suicide and responses of significant others. Acta Psychiatr Scand 1986 May;73(5):481-499. [doi: 10.1111/j.1600-0447.1986.tb02715.x] [Medline: $\underline{3751655]}$ 
9. Wasserman D, Thanh HT, Minh DP, Goldstein M, Nordenskiöld A, Wasserman C. Suicidal process, suicidal communication and psychosocial situation of young suicide attempters in a rural Vietnamese community. World Psychiatry 2008 Feb;7(1):47-53 [FREE Full text] [doi: 10.1002/j.2051-5545.2008.tb00152.x] [Medline: 18458785 ]

10. O'Dea B, Wan S, Batterham PJ, Calear AL, Paris C, Christensen H. Detecting suicidality on Twitter. Internet Interv 2015 May;2(2):183-188. [doi: 10.1016/j.invent.2015.03.005]

11. Czyz EK, Horwitz AG, Eisenberg D, Kramer A, King CA. Self-reported barriers to professional help seeking among college students at elevated risk for suicide. J Am Coll Health 2013;61(7):398-406 [FREE Full text] [doi: 10.1080/07448481.2013.820731] [Medline: 24010494]

12. Kleiman EM, Turner BJ, Fedor S, Beale EE, Picard RW, Huffman JC, et al. Digital phenotyping of suicidal thoughts. Depress Anxiety 2018 Jul;35(7):601-608. [doi: 10.1002/da.22730] [Medline: 29637663]

13. Torous J, Larsen ME, Depp C, Cosco TD, Barnett I, Nock MK, et al. Smartphones, sensors, and machine learning to advance real-time prediction and interventions for suicide prevention: a review of current progress and next steps. Curr Psychiatry Rep 2018 Jun 28;20(7):51. [doi: 10.1007/s11920-018-0914-y] [Medline: 29956120]

14. Shiffman S, Stone AA, Hufford MR. Ecological momentary assessment. Annu Rev Clin Psychol 2008;4:1-32. [doi: 10.1146/annurev.clinpsy.3.022806.091415] [Medline: 18509902 ]

15. Kleiman EM, Nock MK. Real-time assessment of suicidal thoughts and behaviors. Curr Opin Psychol 2018 Aug;22:33-37. [doi: 10.1016/j.copsyc.2017.07.026] [Medline: $\underline{30122275]}$

16. Bidargaddi N, Musiat P, Makinen V, Ermes M, Schrader G, Licinio J. Digital footprints: facilitating large-scale environmental psychiatric research in naturalistic settings through data from everyday technologies. Mol Psychiatry 2017 Feb;22(2):164-169 [FREE Full text] [doi: 10.1038/mp.2016.224] [Medline: 27922603]

17. Onnela J, Rauch SL. Harnessing smartphone-based digital phenotyping to enhance behavioral and mental health. Neuropsychopharmacology 2016 Jun;41(7):1691-1696 [FREE Full text] [doi: 10.1038/npp.2016.7] [Medline: 26818126]

18. Torous J, Onnela J, Keshavan M. New dimensions and new tools to realize the potential of RDoC: digital phenotyping via smartphones and connected devices. Transl Psychiatry 2017 Mar 7;7(3):e1053 [FREE Full text] [doi: 10.1038/tp.2017.25] [Medline: 28267146]

19. Eysenbach G. Infodemiology and infoveillance: framework for an emerging set of public health informatics methods to analyze search, communication and publication behavior on the internet. J Med Internet Res 2009 Mar 27;11(1):e11 [FREE Full text] [doi: 10.2196/jmir.1157] [Medline: 19329408]

20. Eysenbach G. Infodemiology and infoveillance tracking online health information and cyberbehavior for public health. Am J Prev Med 2011 May;40(5 Suppl 2):S154-S158. [doi: 10.1016/j.amepre.2011.02.006] [Medline: 21521589]

21. Tovar D, Cornejo E, Xanthopoulos P, Guarracino M, Pardalos P. Data mining in psychiatric research. Methods Mol Biol 2012;829:593-603. [doi: 10.1007/978-1-61779-458-2 37] [Medline: 22231840]

22. Ruder TD, Hatch GM, Ampanozi G, Thali MJ, Fischer N. Suicide announcement on Facebook. Crisis 2011;32(5):280-282. [doi: 10.1027/0227-5910/a000086] [Medline: 21940257]

23. Luxton DD, June JD, Fairall JM. Social media and suicide: a public health perspective. Am J Public Health 2012 May;102(Suppl 2):S195-S200. [doi: 10.2105/AJPH.2011.300608] [Medline: 22401525]

24. Jashinsky J, Burton SH, Hanson CL, West J, Giraud-Carrier C, Barnes MD, et al. Tracking suicide risk factors through Twitter in the US. Crisis 2014;35(1):51-59. [doi: 10.1027/0227-5910/a000234] [Medline: 24121153]

25. O'Dea B, Larsen ME, Batterham PJ, Calear AL, Christensen H. A linguistic analysis of suicide-related Twitter posts. Crisis 2017 Sep;38(5):319-329. [doi: 10.1027/0227-5910/a000443] [Medline: 28228065]

26. Burnap P, Colombo G, Amery R, Hodorog A, Scourfield J. Multi-class machine classification of suicide-related communication on Twitter. Online Soc Netw Media 2017 Aug;2:32-44 [FREE Full text] [doi: 10.1016/j.osnem.2017.08.001] [Medline: 29278258]

27. Guan L, Hao B, Cheng Q, Yip PS, Zhu T. Identifying Chinese microblog users with high suicide probability using internet-based profile and linguistic features: classification model. JMIR Ment Health 2015;2(2):e17 [FREE Full text] [doi: 10.2196/mental.4227] [Medline: 26543921]

28. Beiwinkel T, Kindermann S, Maier A, Kerl C, Moock J, Barbian G, et al. Using smartphones to monitor bipolar disorder symptoms: a pilot study. JMIR Ment Health 2016 Jan 6;3(1):e2 [FREE Full text] [doi: 10.2196/mental.4560] [Medline: 26740354]

29. Pestian JP, Sorter M, Connolly B, Cohen KB, McCullumsmith C, Gee JT, Research Group ST. A machine learning approach to identifying the thought markers of suicidal subjects: a prospective multicenter trial. Suicide Life Threat Behav 2017 Feb;47(1):112-121. [doi: 10.1111/sltb.12312] [Medline: 27813129]

30. Kessler RC, Hwang I, Hoffmire CA, McCarthy JF, Petukhova MV, Rosellini AJ, et al. Developing a practical suicide risk prediction model for targeting high-risk patients in the veterans health administration. Int J Methods Psychiatr Res 2017 Sep;26(3):- [FREE Full text] [doi: 10.1002/mpr.1575] [Medline: 28675617]

31. Morales S, Barros J, Echávarri O, García F, Osses A, Moya C, et al. Acute mental discomfort associated with suicide behavior in a clinical sample of patients with affective disorders: ascertaining critical variables using artificial intelligence tools. Front Psychiatry 2017;8:7 [FREE Full text] [doi: 10.3389/fpsyt.2017.00007] [Medline: 28210230] 
32. Passos IC, Mwangi B, Cao B, Hamilton JE, Wu M, Zhang XY, et al. Identifying a clinical signature of suicidality among patients with mood disorders: a pilot study using a machine learning approach. J Affect Disord 2016 Mar 15;193:109-116 [FREE Full text] [doi: 10.1016/j.jad.2015.12.066] [Medline: 26773901]

33. Delgado-Gomez D, Blasco-Fontecilla H, Sukno F, Ramos-Plasencia MS, Baca-Garcia E. Suicide attempters classification: toward predictive models of suicidal behavior. Neurocomputing 2012 Sep;92:3-8. [doi: 10.1016/j.neucom.2011.08.033]

34. Simon GE, Johnson E, Lawrence JM, Rossom RC, Ahmedani B, Lynch FL, et al. Predicting suicide attempts and suicide deaths following outpatient visits using electronic health records. Am J Psychiatry 2018 Oct 1;175(10):951-960 [FREE Full text] [doi: 10.1176/appi.ajp.2018.17101167] [Medline: 29792051]

35. Poulin C, Shiner B, Thompson P, Vepstas L, Young-Xu Y, Goertzel B, et al. Predicting the risk of suicide by analyzing the text of clinical notes. PLoS One 2014;9(1):e85733 [FREE Full text] [doi: 10.1371/journal.pone.0085733] [Medline: 24489669]

36. Thompson P, Bryan C, Poulin C. Predicting Military and Veteran Suicide Risk: Cultural Aspects. In: Proceedings of the Workshop on Computational Linguistics and Clinical Psychology: From Linguistic Signal to Clinical Reality. 2014 Presented at: ACL'14; October 9-12, 2014; Baltimore, Maryland, USA. [doi: 10.3115/v1/w14-3201]

37. Walsh CG, Ribeiro JD, Franklin JC. Predicting risk of suicide attempts over time through machine learning. Clin Psychol Sci 2017 Apr 11;5(3):457-469. [doi: 10.1177/2167702617691560]

38. Posner K, Brown GK, Stanley B, Brent DA, Yershova KV, Oquendo MA, et al. The Columbia-suicide severity rating scale: initial validity and internal consistency findings from three multisite studies with adolescents and adults. Am J Psychiatry 2011 Dec;168(12):1266-1277 [FREE Full text] [doi: 10.1176/appi.ajp.2011.10111704] [Medline: 22193671]

39. US Food and Drug Administration. 2012. Guidance for Industry: Suicidal Ideation and Behavior: Prospective Assessment of Occurrence in Clinical Trials URL: http://www.fda.gov/downloads/Drugs/.../Guidances/UCM225130.pdf [accessed 2017-01-04]

40. Ribeiro JD, Pease JL, Gutierrez PM, Silva C, Bernert RA, Rudd MD, et al. Sleep problems outperform depression and hopelessness as cross-sectional and longitudinal predictors of suicidal ideation and behavior in young adults in the military. J Affect Disord 2012 Feb;136(3):743-750. [doi: 10.1016/j.jad.2011.09.049] [Medline: 22032872]

41. Hutto C, Gilbert E. VADER: A Parsimonious Rule-based Model for Sentiment Analysis of Social Media Text. In: Proceedings of the Eighth International AAAI Conference on Weblogs and Social Media. 2014 Presented at: AAAL-14; June 1-4, 2014; Ann Arbor, MI URL: http://comp.social.gatech.edu/papers/icwsm14.vader.hutto.pdf

42. Guo X, Yin Y, Dong C, Yang G, Zhou G. On the Class Imbalance Problem. In: Proceedings of the Fourth International Conference on Natural Computation. 2008 Presented at: ICNC'08; October 18-20, 2008; Jinan, China URL: http://comp. social.gatech.edu/papers/icwsm14.vader.hutto.pdf [doi: 10.1109/icnc.2008.871]

43. Kohavi R. A Study of Cross-validation and Bootstrap for Accuracy Estimation and Model Selection. In: Proceedings of the 14th International Joint Conference on Artificial intelligence. 1995 Presented at: IJCAI'95; August 20-25, 1995; Montreal, Quebec, Canada URL: http://ai.stanford.edu/ ronnyk/accEst.pdf

44. Hastie T, Tibshirani R, Friedman J. The Elements of Statistical Learning: Data Mining, Inference, and Prediction Second Edition. New York, USA: Springer; 2017.

45. Lever J, Krzywinski M, Altman N. Principal component analysis. Nat Methods 2017 Jul 1;14(7):641-642. [doi: 10.1038/nmeth.4346]

46. Breiman L. Random forests. Mach Learn 2001;45(1):5-32. [doi: 10.1023/A:1010933404324]

47. Louppe G, Wehenkel L, Sutera A, Geurts P. Understanding Variable Importances in Forests of Randomized Trees. In: Proceedings of the 26th International Conference on Neural Information Processing Systems. 2013 Presented at: NIPS'13; December 5-13, 2013; Lake Tahoe, Nevada URL: https://dl.acm.org/doi/abs/10.5555/2999611.2999660

48. Biau G. Analysis of a random forests model. J Mach Learn Res 2012;13:1063-1095. [doi: 10.1007/978-1-4419-9326-7_5]

49. Hearst M, Dumais S, Osuna E, Platt J, Scholkopf B. Support vector machines. IEEE Intell Syst Their Appl 1998 Jul 10;13(4):18-28 [FREE Full text] [doi: 10.1109/5254.708428]

50. Cho C, Lee T, Kim M, In HP, Kim L, Lee H. Mood prediction of patients with mood disorders by machine learning using passive digital phenotypes based on the circadian rhythm: prospective observational cohort study. J Med Internet Res 2019 Apr 17;21(4):e11029 [FREE Full text] [doi: 10.2196/11029] [Medline: 30994461]

51. Krystal AD. Psychiatric disorders and sleep. Neurol Clin 2012 Nov;30(4):1389-1413 [FREE Full text] [doi: 10.1016/j.ncl.2012.08.018] [Medline: 23099143 ]

52. Lampinen P, Heikkinen R, Kauppinen M, Heikkinen E. Activity as a predictor of mental well-being among older adults. Aging Ment Health 2006 Sep;10(5):454-466. [doi: 10.1080/13607860600640962] [Medline: 16938681]

53. Sheaves B, Porcheret K, Tsanas A, Espie CA, Foster RG, Freeman D, et al. Insomnia, nightmares, and chronotype as markers of risk for severe mental illness: results from a student population. Sleep 2016 Jan 1;39(1):173-181 [FREE Full text] [doi: 10.5665/sleep.5342] [Medline: 26350467]

54. Domingos P. A Unified Bias-Variance Decomposition for Zero-One and Squared Loss. In: Proceedings of the Seventeenth National Conference on Artificial Intelligence and Twelfth Conference on Innovative Applications of Artificial Intelligence. 2000 Presented at: AAAI'00; October 2, 2000; Austin, Texas URL: https://www.researchgate.net/publication/ 2851463 A Unified Bias-Variance Decomposition for Zero-One and Squared Loss 
55. Geman S, Bienenstock E, Doursat R. Neural networks and the bias/variance dilemma. Neural Comput 1992 Jan;4(1):1-58. [doi: $10.1162 /$ neco.1992.4.1.1]

56. Belkin M, Hsu D, Ma S, Mandal S. Reconciling modern machine-learning practice and the classical bias-variance trade-off. Proc Natl Acad Sci U S A 2019 Aug 6;116(32):15849-15854 [FREE Full text] [doi: 10.1073/pnas.1903070116] [Medline: $\underline{31341078]}$

57. Cover T, Hart P. Nearest neighbor pattern classification. IEEE Trans Inform Theory 1967 Jan;13(1):21-27. [doi: 10.1109/TIT.1967.1053964]

58. Alpaydin E. Combined 5 x 2 cv F test for comparing supervised classification learning algorithms. Neural Comput 1999 Nov 15;11(8):1885-1892. [doi: 10.1162/089976699300016007] [Medline: $\underline{10578036}$ ]

59. Dietterich TG. Approximate statistical tests for comparing supervised classification learning algorithms. Neural Comput 1998 Sep 15;10(7):1895-1923. [doi: 10.1162/089976698300017197] [Medline: 9744903]

60. Swinson N, Ashim B, Windfuhr K, Kapur N, Appleby L, Shaw J. National confidential inquiry into suicide and homicide by people with mental illness: new directions. Psychiatr Bull 2018 Jan 2;31(5):161-163. [doi: 10.1192/pb.bp.106.011734]

61. Braithwaite SR, Giraud-Carrier C, West J, Barnes MD, Hanson CL. Validating machine learning algorithms for Twitter data against established measures of suicidality. JMIR Ment Health 2016 May 16;3(2):e21 [FREE Full text] [doi: 10.2196/mental.4822] [Medline: 27185366]

62. Larsen ME, Nicholas J, Christensen H. A systematic assessment of smartphone tools for suicide prevention. PLoS One 2016;11(4):e0152285 [FREE Full text] [doi: 10.1371/journal.pone.0152285] [Medline: 27073900]

63. Barak-Corren Y, Castro VM, Javitt S, Hoffnagle AG, Dai Y, Perlis RH, et al. Predicting suicidal behavior from longitudinal electronic health records. Am J Psychiatry 2017 Feb 1;174(2):154-162. [doi: 10.1176/appi.ajp.2016.16010077] [Medline: 27609239]

64. Kessler RC, Warner CH, Ivany C, Petukhova MV, Rose S, Bromet EJ, Army STARRS Collaborators. Predicting suicides after psychiatric hospitalization in US army soldiers: the army study to assess risk and resilience in servicemembers (Army STARRS). JAMA Psychiatry 2015 Jan;72(1):49-57 [FREE Full text] [doi: 10.1001/jamapsychiatry.2014.1754] [Medline: 25390793]

65. Kessler RC, van Loo HM, Wardenaar KJ, Bossarte RM, Brenner LA, Cai T, et al. Testing a machine-learning algorithm to predict the persistence and severity of major depressive disorder from baseline self-reports. Mol Psychiatry 2016 Oct;21(10):1366-1371 [FREE Full text] [doi: 10.1038/mp.2015.198] [Medline: 26728563]

66. Alam M, Cho E, Huh E, Hong C. Cloud Based Mental State Monitoring System for Suicide Risk Reconnaissance Using Wearable Bio-Sensors. In: Proceedings of the 8th International Conference on Ubiquitous Information Management and Communication. 2014 Presented at: ICUIMC'14; January 9-11, 2014; Siem Reap, Cambodia p. 1-6. [doi: 10.1145/2557977.2558020]

67. Figueroa RL, Zeng-Treitler Q, Kandula S, Ngo LH. Predicting sample size required for classification performance. BMC Med Inform Decis Mak 2012 Feb 15;12:8 [FREE Full text] [doi: 10.1186/1472-6947-12-8] [Medline: 22336388]

68. Popovici V, Chen W, Gallas BG, Hatzis C, Shi W, Samuelson FW, et al. Effect of training-sample size and classification difficulty on the accuracy of genomic predictors. Breast Cancer Res 2010;12(1):R5 [FREE Full text] [doi: 10.1186/bcr2468] [Medline: 20064235]

69. Beleites C, Neugebauer U, Bocklitz T, Krafft C, Popp J. Sample size planning for classification models. Anal Chim Acta 2013 Jan 14;760:25-33. [doi: 10.1016/j.aca.2012.11.007] [Medline: 23265730]

70. Sordo M, Zeng Q. On sample size and classification accuracy: a performance comparison. In: Oliveira JL, Maojo V, Martín-Sánchez F, Pereira AS, editors. Biological and Medical Data Analysis: 6th International Symposium, ISBMDA 2005, Aveiro, Portugal, November 10-11, 2005, Proceedings (Lecture Notes in Computer Science (3745)). Berlin, Heidelberg: Springer; 2005.

71. Olff M. Mobile mental health: a challenging research agenda. Eur J Psychotraumatol 2015;6(1):27882 [FREE Full text] [doi: 10.3402/ejpt.v6.27882] [Medline: 25994025]

72. Statista. 2019. Percentage of Households With Mobile Phones in the United Kingdom (UK) From 1996 to 2018 URL: https://www.statista.com/statistics/289167/mobile-phone-penetration-in-the-uk/ [accessed 2019-12-02]

73. Tiffin PA, Paton LW. Rise of the machines? Machine learning approaches and mental health: opportunities and challenges. Br J Psychiatry 2018 Sep;213(3):509-510. [doi: 10.1192/bjp.2018.105] [Medline: 30113285]

\author{
Abbreviations \\ C-SSRS: Columbia-suicide severity rating scale \\ SVM: support vector machine \\ SWiM: Strength Within Me
}


Edited by G Eysenbach; submitted 16.08.19; peer-reviewed by K Fox, K Huckvale; comments to author 30.09.19; revised version received 21.02.20; accepted 29.02.20; published 26.06.20

Please cite as:

Haines-Delmont A, Chahal G, Bruen AJ, Wall A, Khan CT, Sadashiv R, Fearnley D

Testing Suicide Risk Prediction Algorithms Using Phone Measurements With Patients in Acute Mental Health Settings: Feasibility Study

JMIR Mhealth Uhealth 2020;8(6):e15901

URL: https://mhealth.jmir.org/2020/6/e15901

doi: $10.2196 / 15901$

PMID: $\underline{32442152}$

(C)Alina Haines-Delmont, Gurdit Chahal, Ashley Jane Bruen, Abbie Wall, Christina Tara Khan, Ramesh Sadashiv, David Fearnley. Originally published in JMIR mHealth and uHealth (http://mhealth.jmir.org), 26.06.2020. This is an open-access article distributed under the terms of the Creative Commons Attribution License (https://creativecommons.org/licenses/by/4.0/), which permits unrestricted use, distribution, and reproduction in any medium, provided the original work, first published in JMIR mHealth and uHealth, is properly cited. The complete bibliographic information, a link to the original publication on http://mhealth.jmir.org/, as well as this copyright and license information must be included. 